\title{
Etude de l'architecture végétale de 14 variétés améliorées de manioc (Manihot esculenta Crantz) dans le centre de la Côte d'Ivoire
}

\author{
*Bakayoko S.1, 2, Soro D. 1, 2, N'dri Be. ${ }^{1}$, Kouadio K.K.H',3, Tschannen A. ${ }^{2}$, Nindjin C. ${ }^{2}$, Dao D. ${ }^{2}$, Girardin $0^{2}$ \\ 1 Université Jean Lorougnon Guède, Département des Sciences de la Terre, 02 BP 150 Daloa 02, Côte d'Ivoire \\ 2 Centre Suisse de Recherches Scientifiques, 01 BP 1303 Abidjan 01 \\ 3 Université de Cocody (Abidjan), UFR des Sciences de la Terre et des Ressources Minières, Laboratoire de \\ Pédologie et de Géologie appliquée, 22 BP 582 Abidjan 22, Côte d'Ivoire
}

Adresse du principal auteur:*BAKAYOKO Sidiky, Tél: +225 07414183 e-mail : sidiky bakayoko@yahoo.fr Original submitted in on 2nd November 2012. Published online at www.m.elewa.org on 31st January 2013.

\section{RESUME}

Objectif : Cette étude a pour objet de comparer l'architecture végétative de quatorze variétés de manioc (Manihot esculenta Crantz) dans le but d'établir une relation avec l'enherbement ou l'association culturale.

Methodologie et résultat : Ainsi, 13 variétés améliorées et un cultivar local ont été plantés sur sol ferralitique à Bringakro dans le Centre de la Côte d'Ivoire. Pendant la croissance, les variétés à grande hauteur de ramification (TME7 $(74,5 \mathrm{~cm})$, TME419 $(81,8 \mathrm{~cm})$, Anader2 $(53,8 \mathrm{~cm})$ et $98 / 0510(51,3 \mathrm{~cm}))$ ont un nombre de ramifaction faible $(23,10,23$ et 26$)$, une grande hauteur du plant et un grand diamètre de tige avec respectivement pour la hauteur $189 \mathrm{~cm}, 199 \mathrm{~cm}, 191 \mathrm{~cm}$ et $196 \mathrm{~cm}$ et pour le diamètre de tige $2,4 \mathrm{~cm}, 2,7 \mathrm{~cm}$, $2,4 \mathrm{~cm}$ et $3 \mathrm{~cm}$. Par contre, les variétés à ramification précoce $(97 / 4769(27,9 \mathrm{~cm}), 96 / 1632(38 \mathrm{~cm}), 98 / 2132$ $(27,1 \mathrm{~cm}), 98 / 0505(28,3 \mathrm{~cm})$ et $97 / 4763(33,1 \mathrm{~cm}))$ ont une petite hauteur $(158 \mathrm{~cm}, 158 \mathrm{~cm}, 171 \mathrm{~cm}, 168 \mathrm{~cm}$ et $147 \mathrm{~cm}$ ), un grand nombre de ramification et un petit diamètre de tige avec respectivement pour le nombre de ramification 42, 45, 40, 36 et 44 et pour le diamètre de tige 2,1 cm, 2,2 cm, 2,2 cm, $2 \mathrm{~cm}$ et $2 \mathrm{~cm}$.

Conclusion et application : Les clones ramifiés sont appropriés pour lutter efficacement contre la compétition des mauvaises herbes tandis que les variétés à port érigé peuvent être utilisées dans des systèmes de cultures en association.

Mots clés: manioc, hauteur, ramification, diamètre de la tige, Côte d'Ivoire.

\section{ABSTRACT}

Objective: This study aimed at comparing the plant architecture of fourteen varieties of cassava (Manihot esculenta Crantz) order to establish a relationship with weed control or intercropping.

Methodology and results: Thus, 13 improved varieties and a local cultivar were planted on ferrallitic soils in Bringakro in the Centre of Côte d'lvoire. During the crop growth, varieties with high branching height (TME7 $(74,5 \mathrm{~cm})$, TME419 $(81,8 \mathrm{~cm})$, Anader $2(53,8 \mathrm{~cm})$ and $98 / 0510(51,3 \mathrm{~cm}))$ had a low number of branches $(23$, 10,23 and 26). They also had a high height and a large stem diameter with respectively for height $189 \mathrm{~cm}, 199$ $\mathrm{cm}, 191 \mathrm{~cm}$ and $196 \mathrm{~cm}$ and for the stem diameter 2,4 cm, 2,7 cm, 2,4 cm and $3 \mathrm{~cm}$. By cons, varieties with 
early branching ( $97 / 4769(27.9 \mathrm{~cm}), 96 / 1632(38 \mathrm{~cm})$ 98/2132 $(27.1 \mathrm{~cm}), 98 / 0505(28.3 \mathrm{~cm})$ and $97 / 4763(33.1$ $\mathrm{cm})$ ) had a small height $(158 \mathrm{~cm}, 158 \mathrm{~cm}, 171 \mathrm{~cm}, 168 \mathrm{~cm}$ and $147 \mathrm{~cm})$. They also had a large number of branching and a small diameter shaft with respectively for the number of branch 42, 45, 40, 36 and 44 and the rod diameter $2.1 \mathrm{~cm}, 2.2 \mathrm{~cm}, 2.2 \mathrm{~cm}, 2 \mathrm{~cm} \times 2 \mathrm{~cm}$.

Conclusion and application: Branched clones are suitable for effectively fighting against weed competition while erect varieties may be used in intercropping systems.

Keywords: cassava, height, branching, stem diameter, Côte d'Ivoire.

\section{INTRODUCTION}

Le manioc (Manihot exculenta) fait partie des principales plantes à racines et tubercules amylacées cultivées dans le monde, du point de vue production (FAOSTAT, 2010). L'Afrique demeure actuellement le principal fournisseur mondial de racines de manioc. En Côte d'Ivoire, le manioc est la deuxième culture vivrière produite après l'igname avec une production de 2'450'000 tonnes réalisée en 2010. Le manioc possède un appareil aérien à développement sympodial simple. On distingue deux types d'axes. Les axes proleptiques, ou ramifications latérales, sont issus du développement de bourgeons axillaires par levée de la dormance apicale (Raffaillac et Second, 1998). Les axes sylleptiques tirent leur origine de la transformation des méristèmes terminaux en méristèmes floraux. $A$ chaque floraison, deux à trois branches se développent simultanément, donnant un aspect di ou trichotomique. Certaines variétés ne fleurissent pas au cours du cycle cultural, d'autres présentent jusqu'à dix floraisons successives sur la tige au cours d'une seule année de culture. Entre ces deux extrêmes, il existe des formes intermédiaires. Ainsi,

\section{MATERIELS ET METHODES}

Site d'étude : Les essais ont été conduits dans le champ expérimental (altitude $150 \mathrm{~m}, 06^{\circ} 40^{\prime \prime} \mathrm{N}, 05^{\circ} 09^{\prime \prime} \mathrm{W}$ ) du Centre Suisse de Recherches Scientifique, à Bringakro, situé à $180 \mathrm{~km}$ au Nord d'Abidjan (Côte d'Ivoire). La parcelle occupe la pente faible (1 à $2 \%$ ) d'un interfluve. La jachère était composée essentiellement $d^{\prime}$ Imperata cylindrica (Poaceae), de Chromolaena odorata (Asteraceae), avec une absence d'éléments grossiers et par endroit des débris végétaux et des turricules d'Hyperiodrilus africanus (Oligochaeta, Eudrilidae)).

Sols : Les sols sont du type ferrallitique moyennement et faiblement désaturé et sont caractérisés par des teneurs l'architecture végétative peut orienter l'agriculteur en vue d'une gestion optimum. L'intérêt croissant dont bénéficie le manioc pour ses qualités, amène à rechercher des moyens en vue d'accroitre les rendements. Le désherbage est une opération culturale obligatoire qui conditionne étroitement le potentiel de production de la plante. Cette situation constitue une préoccupation majeure pour les populations rurales. La verse et la défoliation du manioc en saison sèche favorisent l'enherbement tardif de la culture. Le sarclo-buttage ne permet pas de désherber sur la ligne de plantation, ce travail indispensable ne peut être que manuel et il nécessite plusieurs journées de main-d'œuvre. En outre, la réduction de la couverture du sol provoque un développement plus important des mauvaises herbes grâce à la lumière disponible au niveau du sol. Dans ces conditions, il semble possible de réduire l'enherbement par l'utilisation de variétés à frondaison précoce. L'étude a ainsi porté sur le taux de débourrage, la hauteur du plant, la hauteur de ramification, le nombre de ramification et le diamètre de la tige des plants de manioc.

élevées en sables fins et une faible épaisseur d'humus. La granulométrie des horizons du sol a varié de la surface $(0-28 \mathrm{~cm})$ à la profondeur $(28-53 \mathrm{~cm})$. Les sables ont été prépondérants en surface et en profondeur (78,9$79,5 \%$ ). Le taux d'argiles (kaolinite surtout) a augmenté progressivement avec la profondeur $(5,2-8,6 \%)$, mais est resté inférieur à celui des limons (15,3-12\%). Le pH du sol a été acide en surface (5) et en profondeur $(5,8)$. Le rapport $\mathrm{C} / \mathrm{N}$ a été normal en surface (12) et a diminué avec la profondeur (8). Le taux de matière organique est resté faible sur l'ensemble de la parcelle et a diminué progressivement en profondeur de 0,89 à 0,58\%. La 
teneur en azote total $(0,045 \%)$ est restée insuffisante sur la parcelle entière. Le phosphore total a été faible pour les deux niveaux. II a été de 146 ppm en surface et 133 ppm en profondeur. La CEC a été très faible et a augmenté de la surface vers la profondeur (2,4 et 2,8 méq $/ 100 \mathrm{~g}$ de sol). Concernant les bases échangeables, $S$ (calcium, magnésium, potassium et sodium), les teneurs ont été faibles $(0,881$ à 0,747 méq/100 g) et très inférieures aux seuils critiques. Le taux de saturation en bases a été moyen (37 à 27\%).

Climat du site : La région se caractérise par un climat équatorial de transition avec deux maxima et deux minima (Avenard et al., 1974), une grande saison des pluies (Mars à Juillet) avec un total moyen de $850 \mathrm{~mm}$ d'eau, une petite saison sèche (Juillet à Août), une petite saison des pluies (Septembre à Octobre) qui a donné environ $185 \mathrm{~mm}$ et une grande saison sèche (Novembre à Février). La pluviométrie moyenne annuelle a été de $1153 \mathrm{~mm}$ de 2002 à 2005 avec une température moyenne de $26^{\circ} \mathrm{C}$, une humidité relative moyenne de $77 \%$ et un rayonnement solaire global mensuel de 394 MJ m².

Matériel végétal: Treize (13) variétés améliorées (96/1632, 97/4763, 97/4769, 97/3200, 97/0162, 98/0510, 98/2132, 98/0505, 98/2101, 98/0002, 98/0581, TME7 et TME419) issues de I'IITA (International Institute of Tropical Agriculture) à lbadan au Nigéria et introduites en Côte d'Ivoire en juin 2002 et un (1) cultivar local témoin (Anader2) ont été utilisés comme matériel de plantation. Dispositif expérimental : L'essai a été mis en place selon un dispositif factoriel («split plot») en 4 répétitions comportant chacune 14 parcelles randomisées. Les

\section{RESULTATS}

Taux de débourrage en 2002 et 2003 : Le taux moyen de débourrage par variété a été élevé avec 96/1632 (96\%), 97/4763 (94\%), 97/4769 (96\%), 97/3200 (82\%), 97/0162 (88\%), 98/0510 (91\%), 98/2132 (95\%), 98/0505 (96\%), 98/2101 (93\%), 98/0002 (96\%), 98/0581 (84\%), TME7 (93\%), TME419 (97\%) et Anader2 (82\%) . Le taux de levée de la variété locale (Anader 2) a été la plus faible (82\%), tandis que la variété améliorée (TME-419) a eu le taux le plus élevé (97\%).

Croissance et vigueur des plants : Les plants ont eu des hauteurs relativement faibles (inférieur à $200 \mathrm{~cm}$ ) (Error! Reference source not found.). Deux nouvelles variétés (TME 419 avec $199 \mathrm{~cm}$ et $98 / 0510$ avec $196 \mathrm{~cm}$ ) ont eu une hauteur moyenne significativement supérieure parcelles avaient une dimension de $25 \mathrm{~m} \mathrm{X} 4 \mathrm{~m}$. A la plantation, des boutures de $15-20 \mathrm{~cm}$ de long ont été insérées de façon oblique sur les lignes, à l'écartement de $1 \times 1 \mathrm{~m}$ (densité de plantation 10000 plants/ha). La plantation a été faite manuellement le 06 juin 2002, puis, à la même date en 2003. La variété distribuée au hasard comprend 16 lignes de $25 \mathrm{~m}$ ( $2 \times 4$ lignes utiles et $2 \times 4$ lignes de bordures) par date de plantation.

\section{Collecte de données}

Levée de dormance et débourrage : Les pieds levés ont été comptés sur les deux lignes centrales par variété et par bloc. Les observations ont débuté 2 semaines après la plantation. Une $2^{\mathrm{e}}$ observation a eu lieu à la $3^{\mathrm{e}}$ semaine, en vue de recenser les éventuelles plantules en retard au niveau de la levée.

Hauteur, taux de couverture et vigueur des plantes: A 6 mois après plantation, la hauteur du plant a été mesurée à l'aide d'une règle graduée. Le nombre de ramifications a été compté, la hauteur de ramifications (longueur entre le sol et la première ramification) a été mesurée à l'aide d'une règle et le diamètre de la tige a été mesuré à l'aide d'un pied à coulisse à 15 mois après plantation. Ceci a été fait dans le but d'évaluer le taux de couverture et la vigueur des plants.

\section{Méthodes statistiques}

Les données ont été soumises à l'analyse de la variance suivant le dispositif expérimental (modèle linéaire avec interactions), par le logiciel d'analyse SAS ${ }^{\circ}$ (SAS Institute Inc, Cary, NC, USA). Lorsque les effets étaient significatifs, les moyennes ont été comparées à l'aide de la méthode Student-Newman-Keuls (Dagnelie, 2003).

$(\mathrm{p}<0,05)$ à la variété TME7 $(189 \mathrm{~cm})$. C'est la variété $98 / 0510$, avec $3 \mathrm{~cm}$ qui a eu un diamètre de tige significativement supérieur à TME7 $(2,4 \mathrm{~cm})$. Plusieurs variétés $(96 / 1632(38 \mathrm{~cm}), 97 / 3200(32,8 \mathrm{~cm}), 98 / 0505$ (28,3 cm), 98/2101 $(39,1 \mathrm{~cm}), 98 / 0002(25,6 \mathrm{~cm}))$ émettent précocement des ramifications par rapport à TME7 (74,5 cm). Les variétés 96/1632, 97/4763, 97/4769, 98/2101 et $98 / 2132$ ont émis beaucoup plus de fourches. Relation entre les caractéristiques phénologiques du manioc

Analyse en Composantes Principales: Les axes principaux expliquent le système de la manière assez forte (33\% de variance pour l'axe 1, $55 \%$ de la variance totale expliquée par le cumul des deux premiers axes et 

manioc

$73 \%$. de la variance totale expliquée par le cumul des trois premiers axes) (Tableau 2).

Tableau 1: Croissance et vigueur des plants de manioc

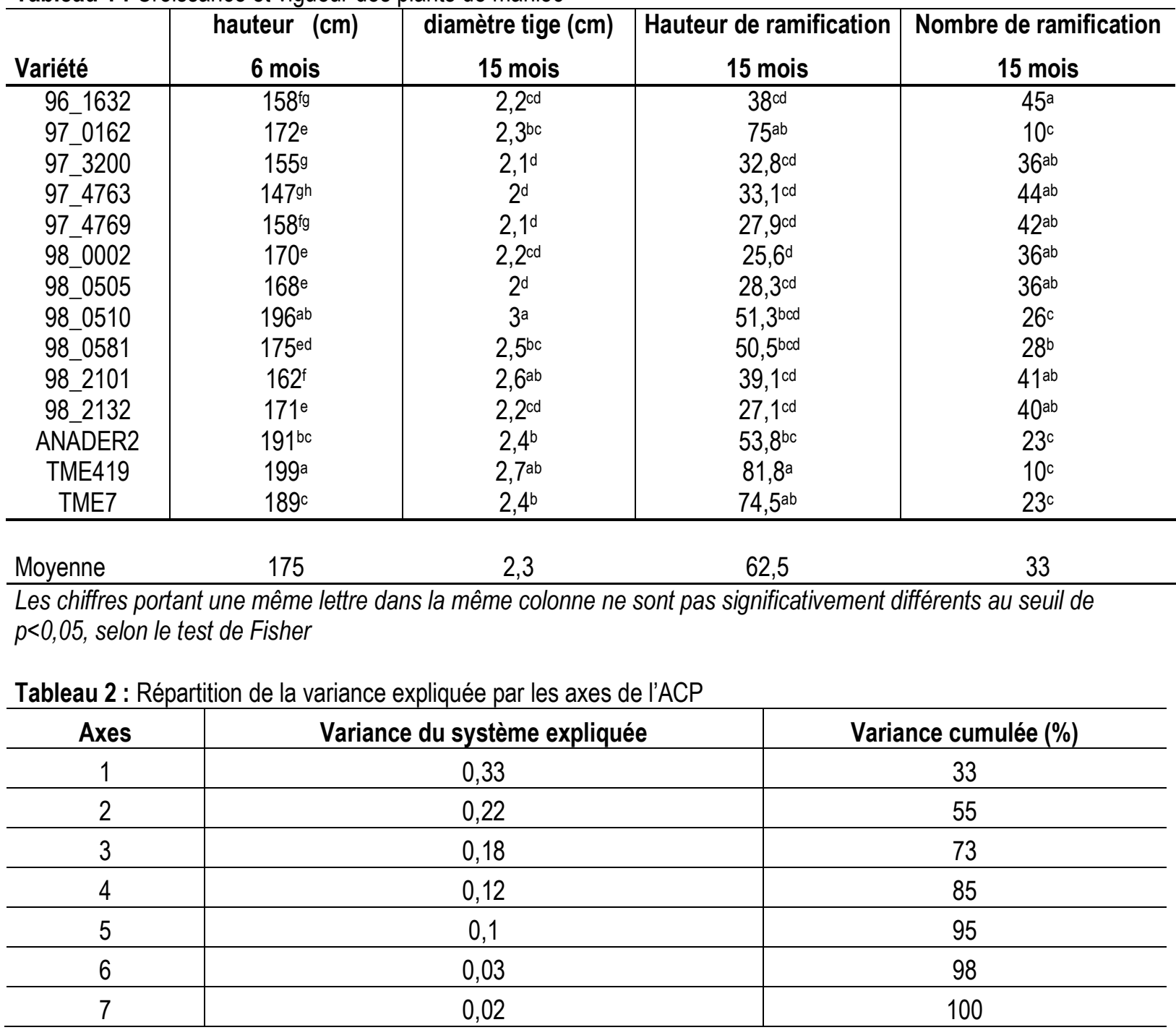

Les individus proches dans les graphiques donnés (axe 1-axe 2) (Figure 1) ont des caractéristiques proches. Les variétés TME7 et TME419 ont eu des tiges vigoureuses et une ramification tardive, tandis que les variétés 96/1632, 98/0505, 97/4769, 97/4763 se sont caractérisées par un grand nombre de fourches. La variété 96/1632 est celle qui a, non seulement la ramification la plus précoce mais, aussi le plus grand nombre de ramifications. II a été observé une corrélation négative entre le nombre de ramification et la hauteur de ramification d'une part et d'autre part entre le nombre de ramification et le diamètre de la tige. II a existé aussi une corrélation positive entre la hauteur du plant et la hauteur de ramification. 
Bakayoko et al...J. Appl. Biosci. 2013. Etude de l'architecture végétale de 14 variétés améliorées de manioc
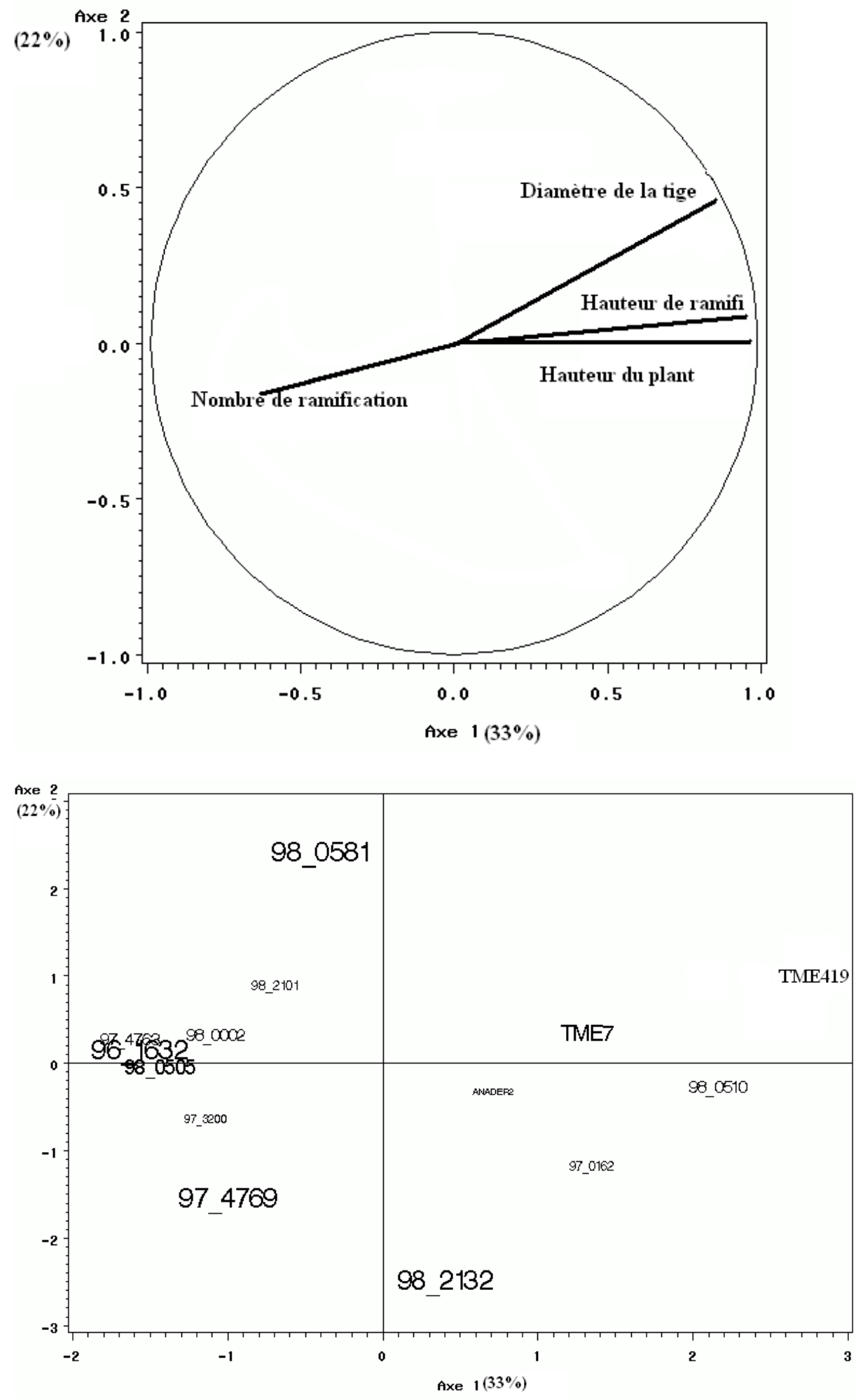

Figure 1: Corrélation entre variétés de manioc et caractéristiques observées 
Classification hiérarchique: L'observation $d u$ dendrogramme permet de sérier les variétés en 2 classes (Figure 2). La classe I, constituée de 9 variétés (98/0581, 98/2101, 98/0002, 98/0505, 98/2132, 97/4763, 97/4769, 97/3200, et 96/1632) s'est caractérisée par un grand nombre de ramifications. Quant à la classe II, composée de 5 variétés (TME7, 98/0510, ANADER2, 97/0162 et TME419), il a présenté des plants de grande hauteur, des tiges à large diamètre, qui ramifient très peu avec une ramification tardive (Error! Reference source not found.)

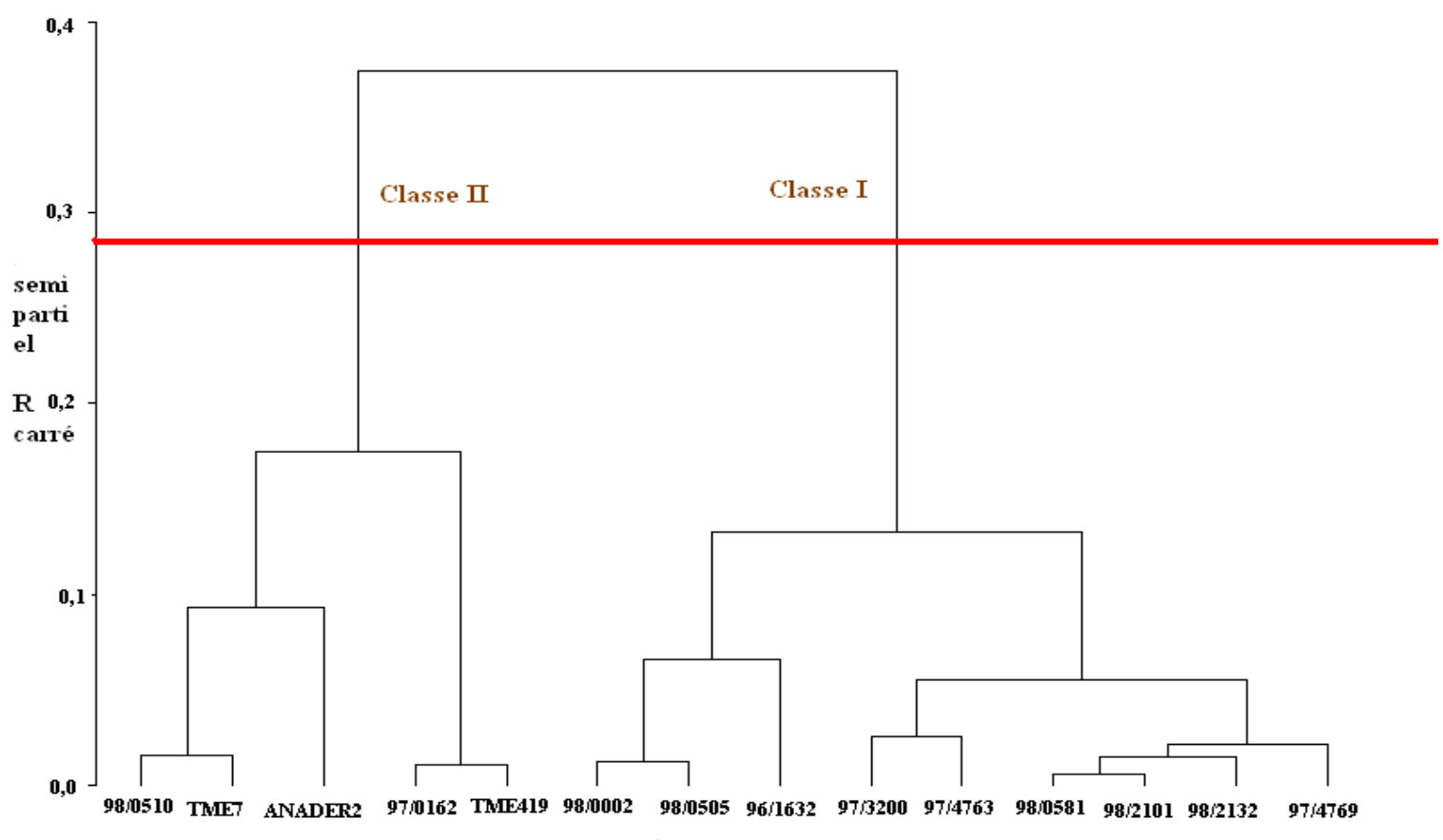

varietes

Figure 2 : Dendrogramme des 14 variétés basées sur les caractéristiques phénologiques

Tableau 3 : Caractéristiques des différentes classes de manioc

Classe I

\begin{tabular}{lccccc}
\hline Variable & $\mathrm{N}$ & moyenne & déviation std & Minimum & Maximum \\
\hline Hauteur du plant & 9 & 166 & 11 & 147 & 181 \\
hauteur de ramification $(\mathrm{cm})$ & 9 & 40,63 & 9,44 & 24,6 & 59,12 \\
diamètre de la tige & 9 & 2,3 & 0,32 & 2 & 2,97 \\
nombre de ramification & 9 & 30,37 & 7,18 & 19,4 & 42,98 \\
\hline
\end{tabular}

Classe II

\begin{tabular}{lccccc}
\hline Variable & $\mathrm{N}$ & moyenne & déviation std & Minimum & Maximum \\
\hline Hauteur du plant & 5 & 189,4 & 10,86 & 172 & 199 \\
hauteur de ramification $(\mathrm{cm})$ & 5 & 59,52 & 3,9 & 44,05 & 74,32 \\
diamètre de la tige & 5 & 2,38 & 0,25 & 2,01 & 2,66 \\
nombre de ramification & 5 & 14,19 & 4,4 & 5,64 & 32,38 \\
\hline
\end{tabular}

\section{DISCUSSION}




\section{Bakayoko et al...J. Appl. Biosci. 2013. Etude de l'architecture végétale de 14 variétés améliorées de manioc}

Nous avons estimé les caractères végétatifs en calculant le diamètre des tiges et les aspects de ramification du manioc dans le cas où ces variables n'évoluent statistiquement plus (MINAGRA \& MRS, 1983). Selon ces auteurs, la masse aérienne peut donc être considérée comme stable vers le quatorzième mois de culture. Avant, la croissance végétative est encore inachevée. ; Après, la stabilité ne peut être considérée comme un arrêt de croissance ; il s'agit plutôt d'un ralentissement, les pertes de feuilles étant compensées par des néoformations. Les résultats de l'ACP ont montré très clairement une variabilité des caractères phénologiques des variétés. En effet, les variétés améliorées résultent de croisement entre des individus qui diffèrent pour un certain nombre de potentialités héréditaires, celles-ci se trouvent réassociées dans l'individu descendant (Rossignol, 1992). Les données obtenues tendent aussi à montrer des variétés ayant des caractéristiques semblables. Ceci pourrait s'expliquer par l'existence de groupes de proximité génétique. Les variétés à hauteurs de ramification élevées ont tendance à présenter des plants de grandes tailles avec des tiges de gros diamètre. Ces plants sont plus vigoureux. Néanmoins, la hauteur

\section{CONCLUSION}

Certains clones se ramifiant très tôt, ont leurs rameaux situés à proximité de la surface du sol et présentent l'avantage d'enrayer le développement des mauvaises herbes. Ceci réduirait le nombre de sarclages impliquant ainsi une baisse du coût de production. D'autres cultivars se ramifiant à une certaine distance du sol, grâce à une

\section{REFERENCES BIBLIOGRAPHIQUES}

Avenard JM, Bonvallot J, Lathan M, Renard-Rugerdil M, Richard J, 1974. Aspect du contact forêt-savane dans le Centre et l'Ouest de la Côte d'Ivoire: étude descriptive, recherches sur le contact forêt-savane en Côte d'Ivoire. ORSTOM (Edition), Paris, 254 pp.

Dagnelie $P, 2003$. Principes d'expérimentation, planification des expériences et analyse de leurs résultats. Grembloux, Les presses agronomiques de Gembloux (Edition), 397 pp.

FAOSTAT (2010). FAO Statistical databases. Italie, Rome, «http://www.fao.org».

Lahai MT, Ekanayake IJ, George JB, 2003. Leaf chlorophyll content and tuberous root yield of cassava in inland valley. African Crop Science Journal. 11: 107-117. peut représenter un handicap en raison de la sensibilité à la verse. En outre, les plants qui sont les plus ramifiés c'est-à-dire qui émettent beaucoup de fourches sont de petites tailles avec des tiges à petit diamètre. Ces résultats sont en accord avec les conclusions de Schulthess et al. (1997). Cette attitude s'explique par le principe même de l'élaboration des fourches. Chez le manioc, le développement des pousses feuillées intervient avec l'offre des matières élaborées (Osiru, 1990). Ces matières élaborées sont partagées entre les pousses et les tubercules, ce qui conduit à une vive concurrence entre ces parties. Par conséquent, conclut cet auteur, un indice foliaire optimum est indispensable pour assurer la meilleure tubérisation possible. Les expérimentations de Lahai et al. (2003) ont aussi montré que l'augmentation de la frondaison s'expliquait par l'accroissement des concentrations des chlorophylles a et ab dans les feuilles. Par ailleurs, le nombre élevé de ramification s'explique par la forte activité photosynthétique des feuilles. Ceci induit une protection des tubercules contre l'insolation permettant ainsi de maintenir les tubercules dans des conditions d'humidité élevée.

frondaison relativement ouverte, offrent l'avantage de permettre l'association avec une culture basse. Les variétés de manioc qui émettent beaucoup de fourches peuvent résoudre rapidement le problème de disponibilité en matériel végétal de plantation

MINAGRA et MRS, 1983. Rapport semestriel d'exécution technique $n^{\circ} 7$, recherches d'accompagnement manioc. IDESSA/SODEPALM (Edition), Bouaké (Côte d'lvoire), 79 pp.

Osiru DSO, 1990. Morphologie et physiologie. In: Le manioc en Afrique tropicale. IIITA (Edition), Nigeria, Ibadan, p.p. 17-27.

Raffaillac, J. P. et Second, G. (1998). Plate-forme Espèces tropicales et méditerranéennes, http://www.brg.prd.fr/brg/ecrans/rgvPi_manioc.ht $\underline{m}$.

Rossignol J-L, 1992. Génétique. MASSON (Edition), France, Paris, $282 \mathrm{pp}$.

Schulthess F, Neuenschwander P, Gounou S, 1997. Multi-trophic interactions in cassava, Manihot esculenta, cropping systems in the subhumid 
Bakayoko et al...J. Appl. Biosci. 2013. Etude de l'architecture végétale de 14 variétés améliorées de manioc

tropics of West Africa. Agriculture Ecosystems \&

Environment 66: 211-222 